\title{
Status of Production of the Superconducting Matching Quadrupoles for the LHC Insertions
}

\author{
R. Ostojic, N. Catalán Lasheras, G. Kirby, J. C. Perez, H. Prin, and W. Venturini Delsolaro
}

\begin{abstract}
Special individually powered superconducting quadrupoles are required for the LHC insertions. These units comprise quadrupole magnets of the MQM and MQY types and range in length from $5.4 \mathrm{~m}$ to $11.4 \mathrm{~m}$. In total, 82 insertion quadrupoles will be assembled at CERN. In this paper we present the advance in construction of the magnets and report on the performance of the first series built units, including power tests and alignment of the cold masses.
\end{abstract}

Index Terms-Insertions, LHC, quadrupoles, superconducting.

\section{INTRODUCTION}

$\mathbf{T}$ HE LHC dispersion suppressors and matching sections contain individually powered quadrupoles, which provide the required tuning of the insertions [1]. These cryomagnets comprise several superconducting quadrupoles of the MQM or MQY types arranged to give the necessary field strength. In the dispersion suppressors the quadrupoles are part of the continuous arc cryostat and are operated at $1.9 \mathrm{~K}$, and although independently powered provide identical cryogenic and powering interfaces to the adjacent main dipoles as in the regular arc cells. Most of the quadrupoles in the matching sections are stand-alone units and are cooled in a static helium bath at $4.5 \mathrm{~K}$. Although in principle simpler, their cryogenic and powering interfaces are determined by the local conditions of each insertion (e.g. slope of the tunnel, interference with injection lines etc.). As a result, the LHC insertion quadrupoles range in length from $5.4 \mathrm{~m}$ to $11.4 \mathrm{~m}$, and comprise up to seven magnetic elements. In total, 82 quadrupoles of 31 different types are in production at CERN. In this paper we present the construction status and report on the performance of the series built magnets and cold masses.

\section{Magnet PROduction}

Two types of quadrupole magnets are used in the LHC insertions: the MQM, which features a $56 \mathrm{~mm}$ coil aperture and is produced in three magnetic lengths $(2.4 \mathrm{~m}, 3.4 \mathrm{~m}$ and $4.8 \mathrm{~m})$, and the $3.4 \mathrm{~m}$ long MQY which has an enlarged $70 \mathrm{~mm}$ coil aperture. Both types of magnets are based on an $8 \mathrm{~mm}$ wide Rutherford-type NbTi cable, such that their nominal currents are $5380 \mathrm{~A}$ and $3600 \mathrm{~A}$, corresponding respectively to field gradients of $200 \mathrm{~T} / \mathrm{m}$ at $1.9 \mathrm{~K}(\mathrm{MQM})$ and $160 \mathrm{~T} / \mathrm{m}$ at $4.5 \mathrm{~K}$ (MQY).

The MQM magnets are produced by Tesla Engineering (England) and the MQY by ACCEL Instruments (Germany). Following the transfer of technology to the firms, the qualification

Manuscript received October 3, 2004

The authors are with the Accelerator Technology Department, CERN, 1211 Geneva 23, Switzerland (e-mail: Ranko.Ostojic@cern.ch).

Digital Object Identifier 10.1109/TASC.2005.849503

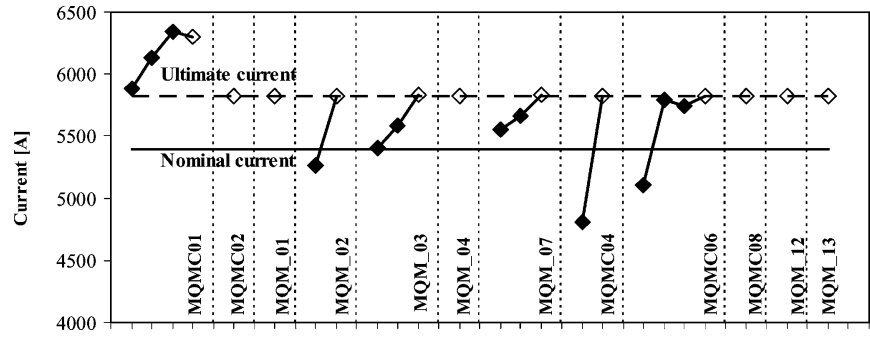

Fig. 1. Quench history of the MQM magnets. Open symbols indicate that no quench occurred. The first pre-series magnet MQMC01 was thoroughly tested and reached the conductor limit in three quenches.

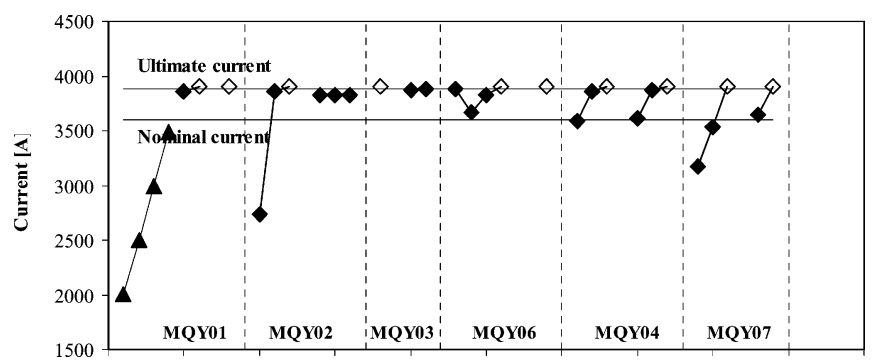

Fig. 2. Quench history of the individually powered MQY apertures. Open symbols indicate that no quench occurred. Triangles correspond to provoked quenches.

of the pre-series units was successfully completed [2], and the production of the series units started. To date, $33 \mathrm{MQM}$ and 10 MQY magnets have been delivered at CERN, corresponding to about $35 \%$ of the total production.

As part of the quality assurance, a series of mechanical, electrical and magnetic tests are performed on the magnets during production. A subset of these tests is repeated at CERN on delivery. As an important quality check, a certain number of magnets are cold tested in the vertical test facility at CERN, which provides a fast feedback to production.

The quench history of the MQM magnets is summarized in Fig. 1. The first pre-series magnet was thoroughly tested and reached the conductor limit in a few quenches. As the magnet did not retrain after a thermal cycle, all other magnets were trained in one cool-down only. All magnets reached the ultimate current in the LHC, corresponding to a field of $9 \mathrm{~T}$ in the main dipoles, in two training quenches or less, about half of the magnets without any training at all.

The results of quench training of the MQY magnets are shown Fig. 2. Due to the large stored energy, each aperture was trained separately. In addition, several quenches were provoked in the first magnet to test the safety of the cryogenic system. All magnets reached the ultimate current in 2-3 quenches. After training, the apertures were powered in series for magnetic field measurements. 

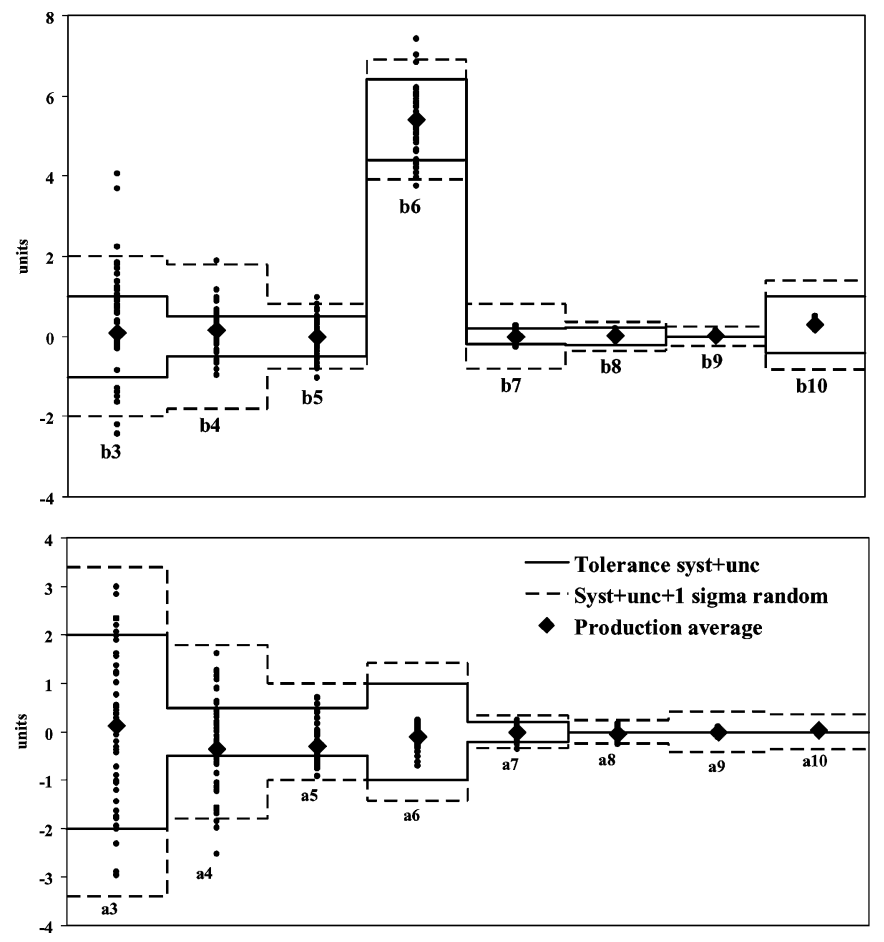

Fig. 3. Normal and skew field multipoles of the MQM magnets (62 apertures). Target limits are indicated by the full and dashed lines.

The magnetic field in all magnets was measured at room temperature before delivery using the CERN supplied magnetic measuring benches. Most of the magnets tested for quench training were also measured cold. The warm-cold correlations obtained indicated that the offsets are small. For production follow-up, we have therefore assumed that the offsets can be neglected, so that the warm magnetic measurements can be directly compared with the specified targets for the field multipoles.

The field quality of the MQM magnets is shown in Fig. 3. The full lines in the figures correspond to the limit on the production average (systematic error plus uncertainty), while the dashed lines correspond to a one-sigma deviation around these average limits. As can be seen, the average value of the MQM multipoles is within the target limits and only a few magnets have multipoles outside the one-sigma boundary. The field quality of MQY magnets is also within specifications [3].

\section{AsSEMbly of Cold MASSES}

The insertion quadrupoles range in length from $5.4 \mathrm{~m}$ to $11.4 \mathrm{~m}$, as shown in Fig. 4. More than half of them contain two magnets connected in series, either of the MQY type, or a combination of MQM magnets of different lengths. In all cases, however, the design principle is the same.

The main structural elements of the cold mass assembly, Fig. 5, are the two half-shells which serve for positioning of the various magnets (quadrupoles and orbit correctors), provide the rigidity for their alignment and serve as a helium pressure vessel. The vessel is closed with two end-domes, which also support the elements required for interconnecting the string of LHC cryo-magnets. In particular, the main $13 \mathrm{kA}$ electrical bus-bars and $1.9 \mathrm{~K}$ helium line are guided by the end-domes,

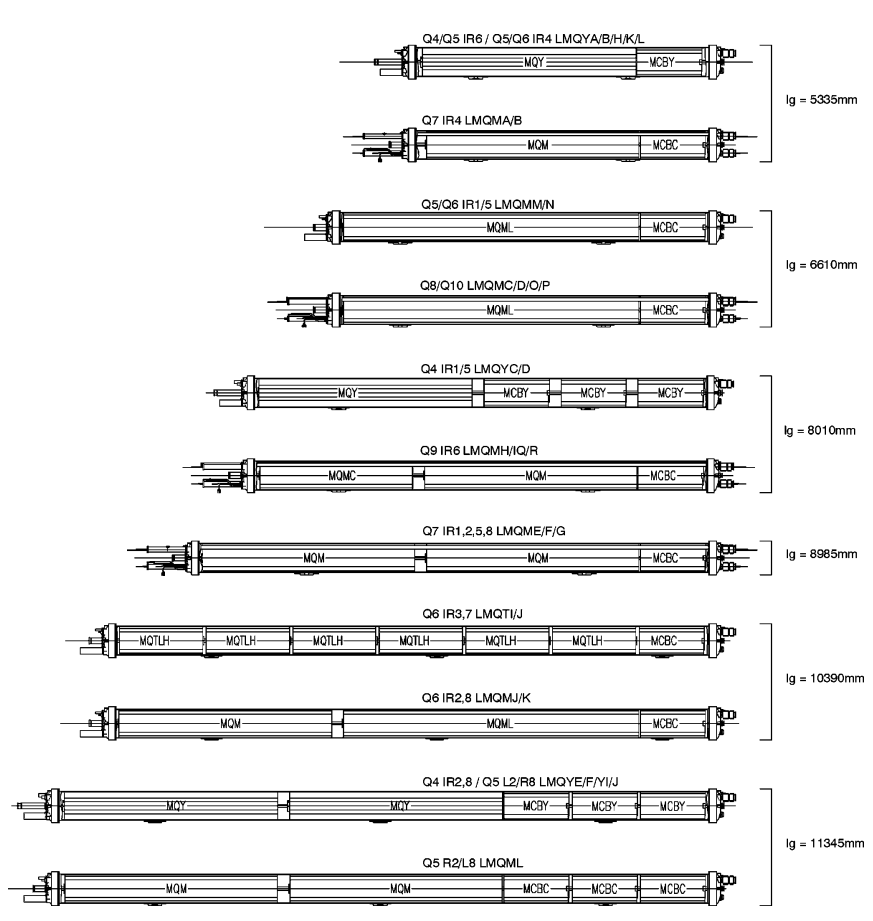

Fig. 4. Types and composition of the LHC insertion quadrupoles.

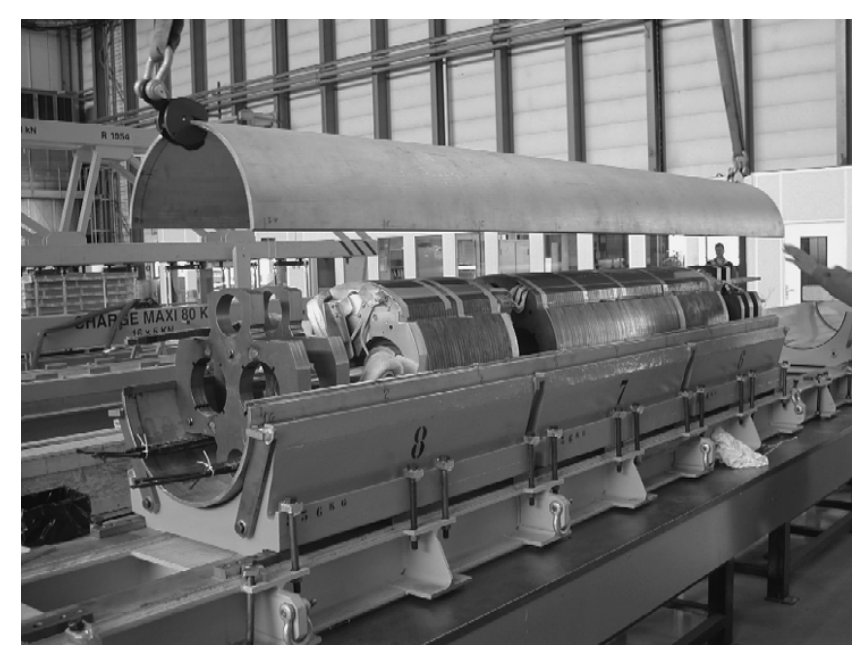

Fig. 5. Insertion quadrupole cold mass before welding of the half shells, showing the magnets and other elements of the assembly.

and the beam position monitors (BPM) and beam vacuum interconnects are precisely positioned with the end-domes as reference. Following the experience gained in the assembly of the pre-series cold mass [4], eight cold masses have been completed in the first half of 2004, and another ten are in different stages of assembly. The cryostating of two cold masses has been completed and they are presently cold tested in the SM18 facility at CERN.

\section{A. Sorting of Magnets}

The optics of the LHC insertions is such that most quadrupoles in the matching sections see transverse beam sizes which are significantly larger than in the arcs and dispersion suppressors. For this reason, and in view of improving the field quality of a subset of cold masses, the magnets are 
assigned to a particular cold mass on the basis of their field quality measured during production.

On the basis of the data for the magnets at CERN and those currently in production, a combination of magnets for a given cold mass is selected such that the resulting field quality is within the full-line limits in Fig. 3. The number of combinations is limited by the available magnets at the time of cold mass assembly. If there is no satisfactory combination, a change in assembly order is introduced, so that magnets manufactured within 2-3 months can be considered for sorting. Typically this involves a choice from a subset of 4-6 magnets. In addition, feedback is given to the companies for the best position of collared apertures in a magnet. The result of this procedure is that the random errors of low order field multipoles $\left(b_{3}, a_{3}, b_{4}, a_{4}, b_{5}\right)$ are reduced by as much as a factor of two. The change in the average values and in the random errors of higher order multipoles is small.

\section{B. Production Experience}

The assembly of the cold masses involves several phases starting with pre-alignment of the magnets, followed by their assembly and alignment in the half-shell, after which electrical connections are performed. The cold mass is then closed and the longitudinal weld completed in the welding press. The final phase is the mounting and welding of the end-domes and completion of the extremities of the beam tubes and cryogenic pipes. The design of components and the assembly procedures and associated tooling were qualified on two prototypes and a pre-series cold mass [4].

The main effort in the initial phase of the series production was focused on resolving the remaining technical problems, streamlining the activities and improving the tooling in those areas which were identified to be on the critical path. In addition, the production team was reinforced and additional training provided.

All operations leading to the welding of the half-shells, in particular the preparation of magnets and work on electrical connections were quickly mastered and the rate of magnet assembly substantially increased. The operations remain nevertheless delicate, and a number of intermediate insulation checks were introduced to avoid time consuming rework. The longitudinal welding, based on the semi-automatic MIG process, Fig. 6, has proven to be very reliable. Simple tooling for placing the top half-shell was developed, so that even the longest shells (12.4 m) are in optimal configuration for the root pass. The welding is now mastered fully, and a cold mass is typically welded in one working day.

The closure of the cold masses remains the most labor intensive and delicate activity. In order to increase the throughput, two assembly benches were fully equipped, Fig. 7, and are used in parallel for any combination of cold masses. The very tight tolerances for the cold mass geometry require precise positioning of the orbital cutting machine, Fig. 8, before the end-domes are mounted and welded. In addition, the extremities of the beam tubes and cryogenic piping must be positioned with utmost care, Fig. 9. All geometrical measurements are performed using an LTD500 laser tracker. Although this precision

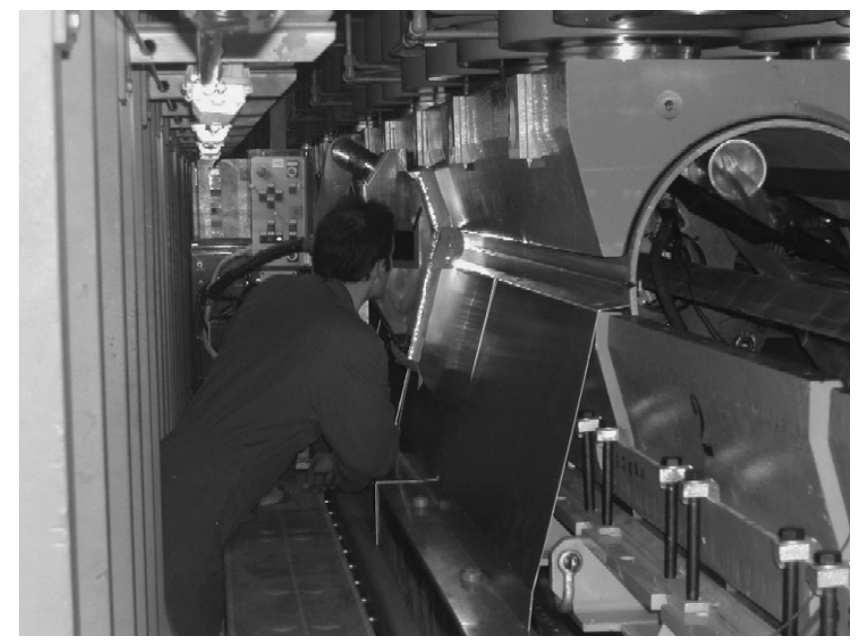

Fig. 6. Longitudinal welding of the half shells in the $18-\mathrm{m}$ welding press.

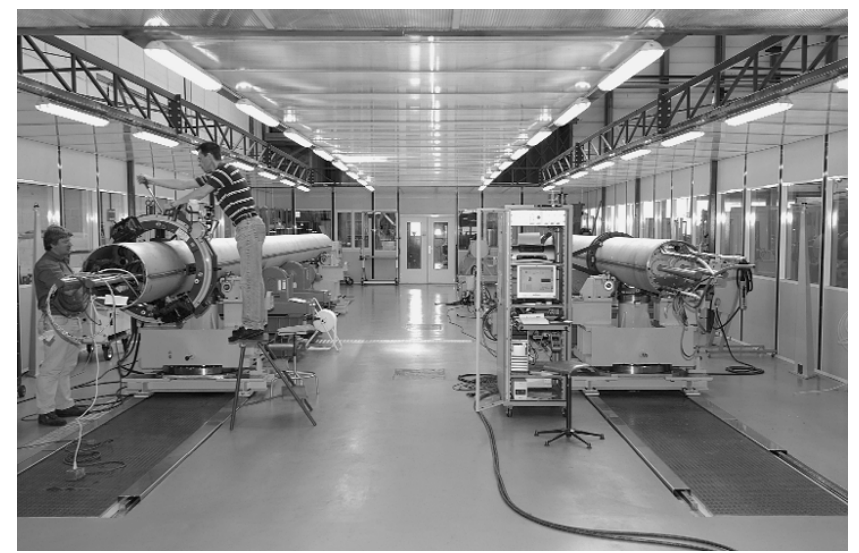

Fig. 7. Welding of end-domes and geometrical verification of the cold masses are performed on two alignment benches in the finishing area.

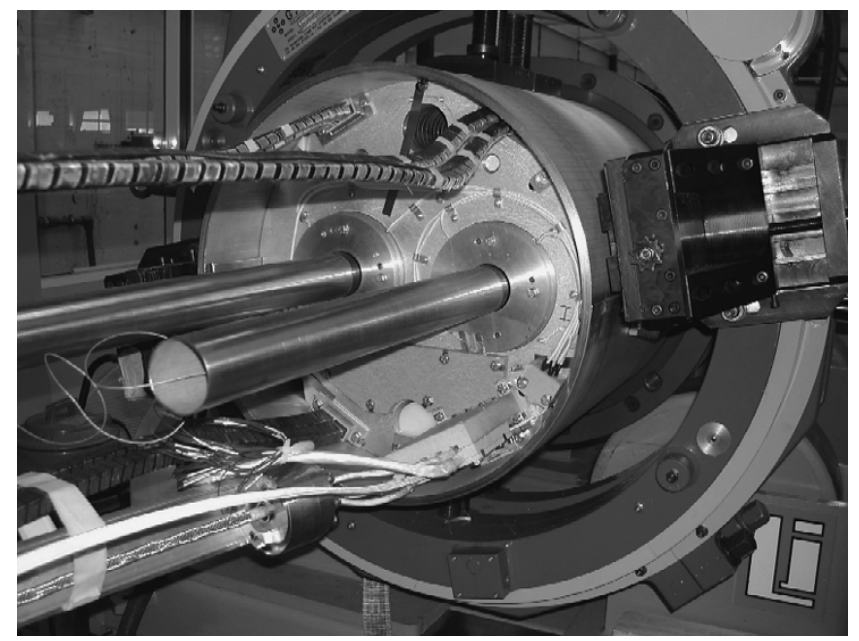

Fig. 8. Preparation of the cold mass lead end for welding of the end-dome.

instrument is adapted to industrial environment, the measurements remain delicate, and frequent checks and calibrations are necessary.

As a result of the improvements in the assembly techniques and procedures, as well as of the gain in efficiency due to the increased experience of the team, the initially planned production 


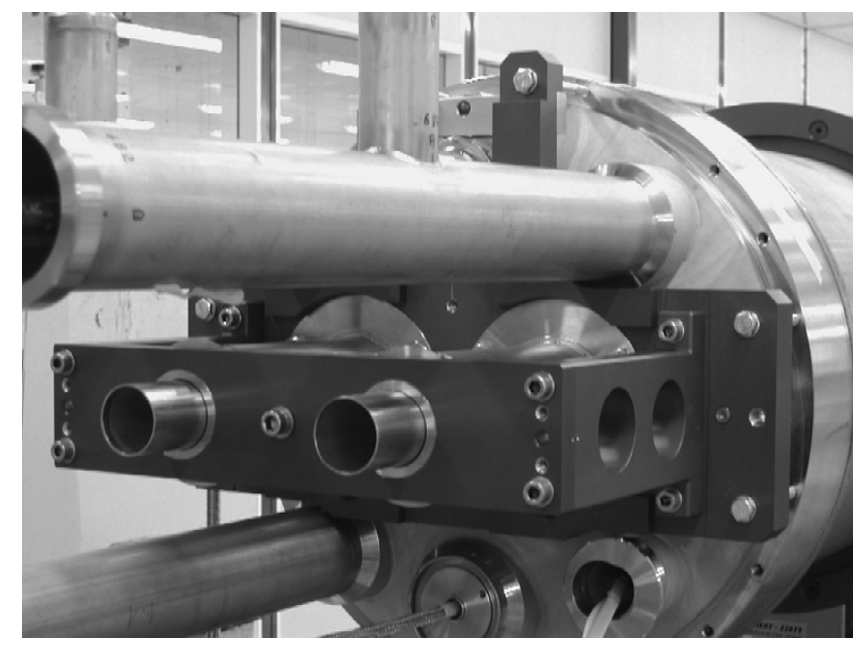

Fig. 9. Positioning of the BPM supports on the lead-end of the quadrupole.
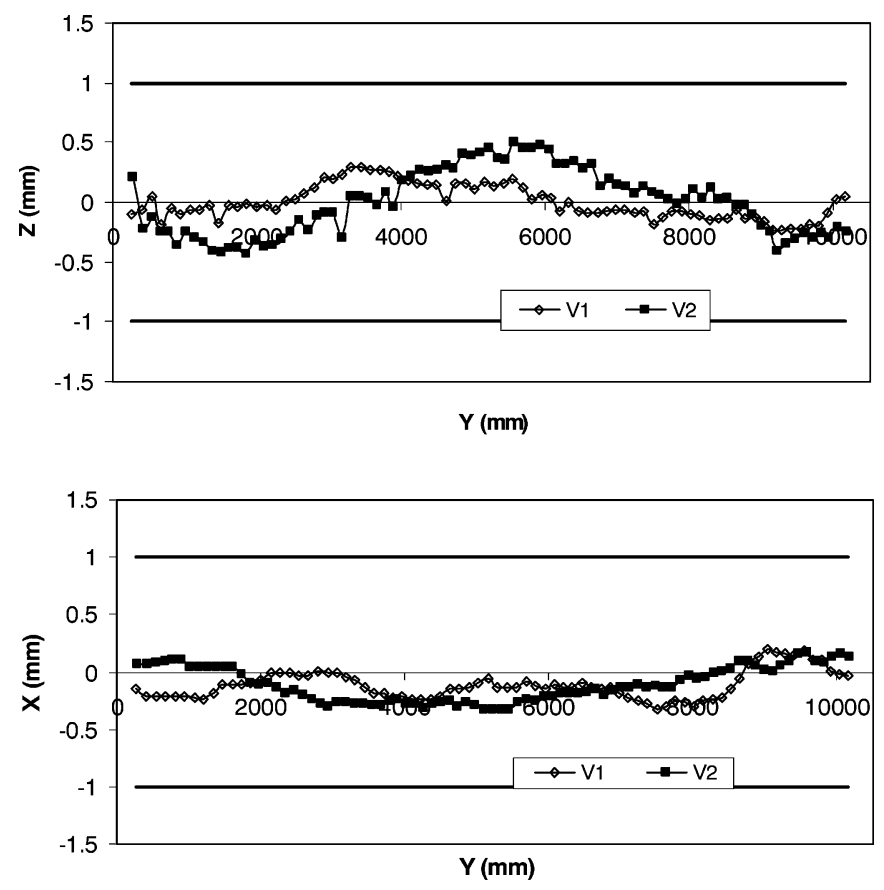

Fig. 10. Alignment of the beam tubes V1 and V2 in the horizontal and vertical planes in the $10.4 \mathrm{~m}$ long Q6 cold mass. The tolerance is $\pm 1 \mathrm{~mm}$.

rate of two cold masses per month was achieved by mid-2004 after three months of series production. Further streamlining is possible, in particular with the better supply and build-up of a stock of critical magnets and other components, and it is expected that a production rate will be further increased by the end of 2004. The production of insertion quadrupoles is planned to be completed by mid- 2006 .

\section{Alignment of the Cold Masses}

Appropriate tooling and maximum care has been taken to achieve the tight alignment tolerances of the cold masses. A typical result is shown in Fig. 10, where the alignment of the 10.4 $\mathrm{m}$ long Q6 cold mass is shown after welding of the end-domes. The measurements are made by tracking an auto-centring mole through the beam tubes, and are reported in the reference system linked to the theoretical position of the beam tubes. These measurements agree remarkably well with those made during production based on the position of the yoke alignment flats, which are used for the alignment of the magnets in the half-shell.

The alignment results obtained on the eight completed cold masses indicate that the relative alignment of the magnets is better than $0.2 \mathrm{~mm}$. The alignment of the cold bores is better than $0.6 \mathrm{~mm}$, and the end domes are within $0.5 \mathrm{~mm}$ from their theoretical position.

\section{CONCLUSIONS}

Following the qualification of the pre-series magnets, the production of the LHC insertion quadrupoles has reached a steady rate of 5 magnets per month, and about $35 \%$ of the production has been delivered. The field quality of the magnets, measured systematically during production, is stable and within specifications. All magnets tested cold, about half of the production, reached the ultimate current in the LHC in less than two training quenches, half of them without any training at all.

The series production of insertion quadrupole cold masses has started beginning of 2004 at CERN. The assembly tooling was refined and the procedures streamlined, ensuring high production quality and increasing the production rate. The production will be completed by mid-2006.

\section{ACKNOWLEDGMENT}

The authors would like to thank the assembly team involved in the quadrupole production and the technicians of the LHC vertical test facility for their excellent work.

\section{REFERENCES}

[1] “LHC Design Report,” The LHC Main Ring, CERN-2004-003, vol. 1.

[2] R. Ostojic et al., "Construction and qualification of the pre-series MQM superconducting quadrupoles for the LHC insertions," IEEE Trans. Appl. Supercond., vol. 14, no. 2, pp. 199-202, 2004.

[3] N. Catalán Lasheras et al., "Performance of the superconducting matching quadrupoles for the LHC insertions," in Proc. EPAC, Lucerne, Jul. 2004.

[4] R. Ostojic et al., "Design and assembly of the pre-series quadrupoles for the LHC insertions," IEEE Trans. Appl. Supercond., vol. 14, no. 2, pp. 203-206, 2004. 